\title{
The Professional Development Plan Process in an Educational Setting: The Need for Revision
}

\section{Rick Stalnaker}

Department of Social Studies, South Iredell High School, USA

*Corresponding author: Rick Stalnaker, Department of Social Studies, South Iredell High School, USA, Tel: 7046812221; E-mail: rstalnaker@iss.k12.nc.us

Received date: Nov 21, 2018; Accepted date: Dec 05, 2018; Published date: Dec 08, 2018

Copyright: () 2018 Stalnaker R. This is an open-access article distributed under the terms of the Creative Commons Attribution License, which permits unrestricted use, distribution, and reproduction in any medium, provided the original author and source are credited.

\author{
Abstract \\ The Professional Development Plan (PDP) is designed to improve an educator's ability in various areas, which \\ may include: \\ - Teacher's demonstration of leadership \\ - Teacher's ability to build a respectful learning environment for students \\ - Teacher's display of knowledge within a content area \\ - Facilitation of learning for students \\ - Reflection on educational practices
}

Keywords: Social; Education; Environment; Communities

\section{Introduction}

As these components are essential for development, the custom of self-assessment and utilization of the process is lacking in effectiveness and does not correlate well with other functional methods of the Professional Learning Communities (PLC) within the educational environment. Some of the fundamental issues with the PDP is that the process serves no structural purpose that would assist in the guidance of new teachers for the establishment of a functional base to assess their skills nor does the process assist in the reflection of best practices. The development plan provides no measure on how to establish a productive, diverse learning environment nor does it guide in the facilitation of learning among students. Due to no gauge or rubric that suggest if the teacher is demonstrating leadership at the school or in the classroom. The concept of evaluating if the teacher is displaying content knowledge in an academic discipline is non-existing as the self-assessment does not focus on content (it is a one size fits all instrument).

\section{Functionality of an Effective Professional Development Plan}

Mizell [1] suggested it is essential that a Professional Development Plan (PDP) be focused on an intensive exertion toward advancement of a teacher's practice and capabilities in an educational environment. Another purpose of the PDP should be to identify intensive improvement approaches that are designed to advance professional competency and student outcomes. The PDP should also address issues concerning professionalism, obligations, and accountability. It may be critical for the PDP to approach ethical expectations.

For the Professional Development Plan (PDP) to be effective, there should be specific guidelines and a precise timetable for the development of a teacher's progress. The PDP should establish supporting components to assist in the identification of standards that teachers may need to improve. However, these standards that build opportunities for improvements should also contain modules to help a teacher reach competency levels. It is necessary for the PDP to be equipped with enhancement contingencies that promote clear expectations about the deviations needed in performance, which should be exhibited in the classroom and school settings [1]. The PDP should have a process that allows guidance and support that can assist the teacher through their reflection procedure concerning best practices. The primary function of the PDP should be implementation of progress in the educational environment.

Mizell [1] suggested that research has indicated that teacher quality and leadership should be the two most important components in boosting student achievement. Adapting the PDP and implementing the plan into the Professional Learning Communities (PLC) could be essential in the educators' assimilation to better assist student learning at elevated levels. The teacher that does not commit to effective professional development may not grow as an educator, and the learners will be the ones that suffer the consequences.

\section{Revising the Professional Development Plan}

The revision of the Professional Development Plan (PDP) should consist of moving away from the current composition of a generic instrument based on self-assessment and toward more cognitive apprenticeship approach that would establish a concentrated apparatus to appraise the educator's professional growth in their specialized academic discipline. A model for the revision of the PDP may include:

- Peer Coaching in conjunction with the PLC driven by PDP data,

- Content focused PDPs associated with extended online PLC's pilot programs,

- Learning modules focused on PDP opportunities for improvements,

- Using PDP data in a teacher collaborative environment,

- Using a blogging approach to improve professional development in PLCs. 


\section{Content Focus on Professional Development Plans}

The Institute of Education Sciences [2] suggested that building a teacher's content knowledge and their overall knowledge associated with content-centered discipline should provide evidence that the PDP focuses on improving the educator's academic discipline knowledge. Most PDP processes are based on commercially accessible methods that are composed of summer institutions, teacher meetings, and coaching during the school year. The Institute of Education Sciences [2] examined research from three educational studies that addressed the question: "Does content-focused teacher professional development work?" The researchers at the Institute of Education Sciences introduce two key points that were determined by the educational studies:

- Accelerated, content-focused PDPs can improve an educator's knowledge and certain factors associated with their practices.

- A PDP that only focuses on improving an educator's content knowledge and practices do not always correlate with student achievement.

In many PDPs the student learning component is overlooked. However, a PDP that focuses on content areas knowledge that would include module training within a Professional Learning Community (PLC) where teachers could work through modules and evaluate the data and collaborate with other teachers could be an efficient approach to develop best practices that could assist in increasing student achievements. The current self-assessment mode of a PDP does not provide module training in areas such as student practical application or direction for using group assignments in a classroom setting. Using an application known as Canvas, teachers could develop modules for enriching content knowledge and share best practices that have improved student performance. These are just examples of how a wellconstructed PDP could focus more on opportunities for improvement for the individual teacher.

Bibbo and d'Erizans [3] suggested that traditional PDPs have run their course as a strategy for development. The researchers contend that future advancements for professional educator relied on a fundamental shift in how we realize learning and the environment by which teachers work with other teachers and students. Collaboration in the content areas that are geared to module training for teachers to gather precise data and distributing those results with other teachers could lead to best practices that are student-centered and may increase achievement. A PDP process that leads to teachers being skilled in their content areas and equipped with knowledge that is concentrated on student-centered best practices should focus on the following elements:

- Distinctive content of subject area knowledge

- Active learning

- Adequate timetable for progress and subject pacing guides

- Feedback and reflection on best practices and summative data

- Collaboration among teachers and students

Bibbo and d'Erizans [3] suggested this process will require educators to amend traditional methods and assimilate the verified characteristics in alternative PDPs. However, the process may be a long-term plan that involves resources, time, revenue, and fixed outcomes.

Vavasseur and MacGregor [4] conducted a mixed-method case study that focused on how professional development among teachers are expedited through professional learning communities (PLC) associated with content centered online practices. The researchers suggested that a key finding in their study revealed that online PLCs could provide educators with a diverse enhanced opportunity to collaborate best practices, discuss barriers, and make new connections with other educators. This process could allow educators to use online module training methods associated with PDPs and increase awareness for best practices on a larger scale across cultures. By using online PLCs with PDP module training, teachers could gain curriculum-based knowledge, establish strong self-efficacy associated with implementing the use of technology, and collaboration on PDP interdisciplinary content-based periods of instruction designed to improve student achievement.

The process of extending academic discipline focused professional development with online professional learning communities (PLC) is associated with a cognitive apprenticeship approach. Kuo et al. [5] suggested that the enhancement process of students' problem-solving aptitude is an essential and challenging issue in a technological driven learning environment. The researchers conducted a study to evaluate the effects of student factors on problem-solving capabilities within a cognitive apprenticeship model. A cognitive apprenticeship model should emphasize the essentials associated with the instrument's (PDP) process by which a teacher of a particular discipline could teach a practice to an apprentice (less experienced teacher) to improve a particular skill. Kuo et al. [5] discovered that learners could improve problem solving skills when a cognitive apprenticeship model is associated with collaborative learning.

\section{Peer-Coaching Utilization within the Professional Learning Community driven by the Professional Development Plan Data}

The peer-coaching approach can serve as an effective tool associated with observations of teachers' content area knowledge. In the classroom, peer-coaches can collect data on how the teacher demonstrates that knowledge and how students interact in the classroom. The professional learning community (PLC) will serve as an apparatus to reflect on the data with other teachers and peer-coaches to improve practices.

Zhang et al. [6] examined peer coaching for teachers associated with online professional learning communities. The researcher suggested that the facilitation of professional development of educators has an opportunity for improvement and could contribute to the overall educational quality of the students. Using an online PLC could be essential in providing professional development opportunities that are linked to interactive activities, peer interactions, and course training. The professional learning environment link to PDPs could assist with teacher interactions associated with problem-solving, sharing best practices, and data connected with student outcomes (what worked and what did not work). For the online PLC to function it should be coordinated with PDPs there should be roles defined in the PLC, such as mentors, coaches, and recorders. The primary aim of the PLC should be to assist teachers with opportunities for improvement. The PDP apparatus should be developed to provide data that reflects teachers' needs. An appraisal of student achievement data should be monitored as practices are implemented [6].

- A cognitive work analysis (CWA), which is a scheme for the design, interpretation, and reflection on a complex sociotechnical approach could be an effective approach to implement in the professional learning communities [7]. The CWA approach may be 
associated with the process of linking the PLC with the PDP. The researcher suggested that a CWA could provide a foundation for environmental interaction that could be associated with higher performance levels. This attribute correlates with the concept of the PLC and PDP driving both teacher and student performances. The CWA method in PLCs and their link to PDPs could address human factors and best practices in a collaborative effort to improve student achievement. Naikar [7] suggested the process should include:

- Work environment analysis

- PLC task analysis

- Best practice analysis

- Student performance and cooperation analysis

- Teacher competence analysis

\section{Exploring Professional Development Programs for Improving Collaboration and Teacher Performance}

Ciampa and Gallagher [8] introduced a unique communication tool for professional development and collaboration in professional learning communities. The researchers suggested blogging, which is an online journal that could be used to share ideas, best practices, and discuss issues in the educational environment. Ciampa and Gallagher [8] implied that blogging could be an appropriate device for a teachers professional development during the collaborative analysis. The researchers used a mixed-method design that involved the collection of quantitative and qualitative data. Ciampa and Gallagher [8] suggested that the results of the study provided evidence that there was both benefits and challenges in using blogs for this purpose. Participants in the mixed-method study implied that the use of blogs for professional development was a essential tool for obtaining feedback on ideas, sharing best practices to improve student performance, improving content knowledge, lesson planning strategies, reflection on practices, and networking with other teachers. However, there were some challenges. Educators need to commit to the process, there must be a sufficient amount of teacher training modules to ensure professional development, and teachers should also have some technical knowledge to interact on the device [8].

Billett and Choy [9] suggested that professional development in the workplace takes on a new perspective and challenges for education. The researchers examined the development of learning in the work environment and the challenges to make the process effective. The researchers discovered that learning in the workplace involved multichannel and complicated factors such as socio-cultural issues and barriers that could impact learning. These factors can be observed in online PLCs and the incorporation of PDPs. Billett and Choy [9] suggested that planning in these areas and extending the constraints of academic disciplines and embrace different cultural sensitivity could lead to a better understanding of the development of the educational environment. Revising the PDP and using it as a tool within PLCs is simply a starting point to improve training for teachers and boost our education system for students.

\section{Conclusion}

A professional development plan that is associated with professional learning communities could be an efficient approach to improve the collaboration among teachers and the development of all teachers. However, there is no room for miscommunication in the overall process. Studies of effective professional development and how it can impact teacher training and student learning can be difficult endeavor to connect. However, if a school makes researched-based decisions about professional development approaches and collaboration and associate these components for enrichment training of teachers and specific student learning outcomes the educational communities may produce positive results.

\section{References}

1. Mizell H (2010) Why Professional Development Matters (1stedn). Oxford, $\mathrm{OH}$ : Learning Forward.

2. Institute of Education Sciences (2016) Does content-focused teacher professional development work? Findings from three institute of education science studies.

3. Bibbo T, D'Erizans R (2014). Professional development that works. Principal Leadership 14: 28-32.

4. Vavasseur C, MacGregor S (2008) Extending content-focused professional development through online communities of practice. Journal of Research on Technology in Education 40: 517-536.

5. Kuo F, Hwang G, Chen SC, Chen SY (2012) A Cognitive Apprenticeship Approach to Facilitating Web-based Collaborative Problem Solving. Educational Technology \& Society 15: 319-331.

6. Zhang S, Liu Q, Wang Q (2017) A study of peer coaching in teachers' online professional learning communities. Universal Access in the Information Society 16: 337-347.

7. Naikar N (2017) Cognitive work analysis: An influential legacy extending beyond human factors and engineering. Applied Ergonomics. 59: 528-540.

8. Ciampa K, Gallagher T (2015) Blogging to enhance in-service teachers' professional learning and development during collaborative inquiry. Educational Technology, Research and Development 63: 883-913.

9. Billett S, Choy S (2013) Learning through work: Emerging perspectives and new challenges. Journal of Workplace Learning 25: 264-276. 